\title{
A Radio-Telemetry System for Navigation in Free-Roaming Rats
}

\author{
Dian ZHANG $^{1, a}$, Wei CHEN ${ }^{1}$, Biao HUANG ${ }^{1}$ \\ ${ }^{1}$ Department of Automation \& Electronic Engineering, Qingdao University of Science and Technology, Qingdao, Shandong, China
}

\begin{abstract}
A multi-channel implantable electrical stimulator is described based on wireless microcontroller CC2430. PWM conversion circuits to DAC and the voltage-controlled current source are designed, the latter of which is needed for electrical stimulator circuits. Through the multi-channel analogy switches, positive and negative pulses on the rat brain areas can be achieved. The positive and negative waves are shown to verify the validity of the circuits.
\end{abstract}

\section{INTRODUCTION}

In recent years in the field of robots, a variety of robots emerge one after another. While they are becoming smarter in intelligence and smaller in size, they cannot walk afar just because of the limited energy the microrobot carries. If the existing small animals can be manipulated, it is possible to solve the problem of inadequate energy carried by micro-robots, thus, a new way is available for rehabilitation of the disabled.

This kind of robots is called animal-robot. Compared with robots or bio-robots, animal-robots have the advantages of efficient energy supply, motion flexibility, mobility and adaptability. Therefore, the relevant research results in the past few years aroused great concern of scholars both at home and abroad. In 2001, Professor Isao Shimoyama, Tokyo University, Japan, fitted cockroach (Robot Roach) with electronic backpack, and through appropriate stimulation the cockroach was able to follow given instructions to turn right or left[1-2]. In 2002, Nature magazine reported that New York State University Medical Center, Professor Chapin implanted electrodes in different brain regions of rats, and through appropriate stimulation the rats could walk along a certain route assumed by human beings[3].In 2004, Professor Atema in Boston University successfully implanted electrodes in the brain of a shark, and he could control the direction of the shark under the water, using the chip implanted in the body of the shark[4]. Since 2002, Wu and Wang[5-6] successfully achieved the transformation of stimulating signals by using wireless remote control system to replace the connecting wires. The two researchers have laid a foundation for the development of animal-robots.

The crux of researching on animal-robots is how to control animals' behavior though the implanted electrodes which can make effective stimulation in some special parts of animal brains. The common method of electronic stimulation is to connect stimulation devices to electrodes

\footnotetext{
a Corresponding author: zhangdian2002@163.com
}

on animals' heads though wires, and then the periodic voltage pulse signals are applied to electrodes for stimulating the brain. However, these wires which seriously restrict the freedom of animals are prone to enlace and brake. The wires can either divert animals' attention or make them suffer from pain. This method cannot achieve the purpose of complete control and the experiment was done under the condition of animals' freedom. The most important problem is that the muscle of animals will be charged and discharged because of its capacitance characteristics, and as time increases the values of animals muscle resistance will be changed. So the control effect will be worse for these reasons.

Now the implantable stimulator circuits which are the research focuses are introduced. And multi-channel implantable stimulator circuits based on current sources are described. Comparing with the electrical nerve stimulation based on voltage source, the current stimulator circuits have some advantages of accurate positioning, small stimulated current, stable values and less pains etc.

\section{Rinciple}

Rats used in physiology study and physiological experiments are chosen as animal-robot objects. The entire implantable multi-channel electrical stimulation of brain circuits consists of power circuit, wireless microcontroller CC2430, and shaping circuit for PWM, $\mathrm{RC}$ filter circuit, voltage-controlled current source and multi-channel analog switches. The I/O ports of CC2430, which make 4 channels of adjustable duty cycle export completely PWM waveform though the shaping circuit. And then the PWM waveform becomes a voltage signal through a second order RC low-pass filter network. The operational amplifier can make the voltage signal become voltage-controlled current source, whose output current size is linear adjustable from $40 \mathrm{uA}$ to $200 \mathrm{uA}$. By matching the appropriate resistor of op amplifier, the 
output current is a fixed value, irrelevant with the load resistance and by multi-channel analog switches the positive and negative pulses to stimulate the rat brain areas can be achieved. The principle of the system is shown in Fig.1. Rats used in physiology study and physiological experiments are chosen as animal-robot objects. The entire implantable multi-channel electrical stimulation of brain circuits consists of power circuit, wireless microcontroller CC2430, shaping circuit for PWM, RC filter circuit, voltage-controlled current source and multi-channel analog switches. The I/O ports of CC2430, which make 4 channels of adjustable duty cycle export completely PWM waveform though the shaping circuit. And then the PWM waveform becomes a voltage signal through a second order RC low-pass filter network. The operational amplifier can make the voltage signal become voltage-controlled current source, whose output current size is linear, adjustable from $40 \mathrm{uA}$ to $200 \mathrm{uA}$. By matching the appropriate resistor of op amplifier, the output current is a fixed value, irrelevant with the load resistance and by multi-channel analog switches the positive and negative pulses to stimulate the rat brain areas can be achieved. The principle of the system is shown in Fig.1.

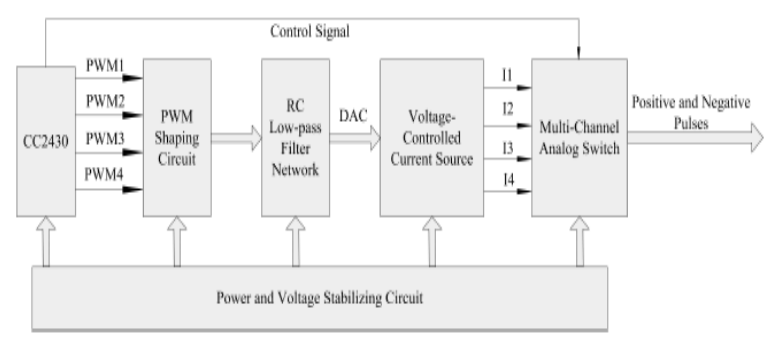

Fig. 1 Principle of implantable multi-channel electrical stimulation

\section{System design}

According to Figure 1, PWM waveform can be given directly from the pins of CC2430. Because the power supply to CC2430 is $+3.3 \mathrm{~V}$, PWM waveform can be achieved by switching the small signal transistor, there is no external benchmark voltage needed after shaping the PWM waveform into voltage single through the second order RC low-pass filter network. According to the fundamental frequency in Equation 2, the value of $\mathrm{R}$ and $\mathrm{C}$ can be set. The cut-off frequency of a low-pass filter in actual circuit is about $1 / 4$ of fundamental frequency. For the reason that CC2430 drive capability is too poor, an external pull-up resistor to drive the next circuit is needed. The circuit that changed PWM to DAC is shown in Fig.2.

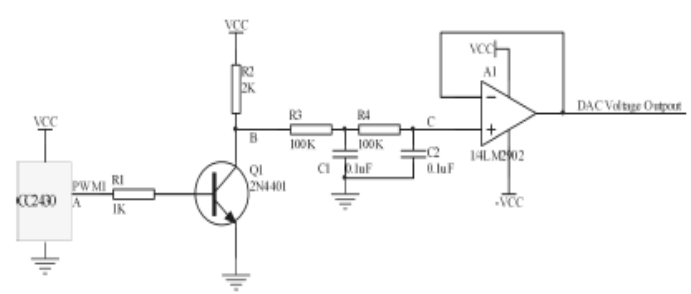

Fig. 2 Circuit changed PWM to DAC

PWM waveform from point A of MCU drives the base of Q1, and Q1, which has little on-resistance and large off-resistance, is the switching transistor 2N4401. It switches according to the duty cycle of PWM. An ideal PWM waveform can be achieved form point $\mathrm{B}, \mathrm{VH}$ is $3.3 \mathrm{~V}$ and $\mathrm{VL}$ is $0 \mathrm{~V}$. After passing the second order RC low-pass filter network, a stable voltage comes from point C. The op amplifier A1 is one channel of LM2902, which includes 4 channels, and a stable voltage can be got when the op-amplifier is working under the following model However, LM2902 power supply is at $3.3 \mathrm{~V}$, the result of the experiment shows that the maximum voltage of $\mathrm{A} 1$ is $+2.3 \mathrm{~V}$, which can meet the requirements. The proof of this issue will be given in the next section.

Studies have shown that the waveform of stimulation pulse is associated with polarity on the release of nerve impulses [7]. And the accumulation of charge will produce permanent injury to animal tissues. To solve the problem, symmetrical bipolar stimulation pulses is designed. Both positive and negative power supply are required to achieve traditional bipolar pulses, thus, multichannel analog switches are applied to achieve bipolar stimulation for the rat brain regions. To be specific, MAX4761A is applied to achieve the positive and negative pulse generation, whose principle is shown in Fig.3.

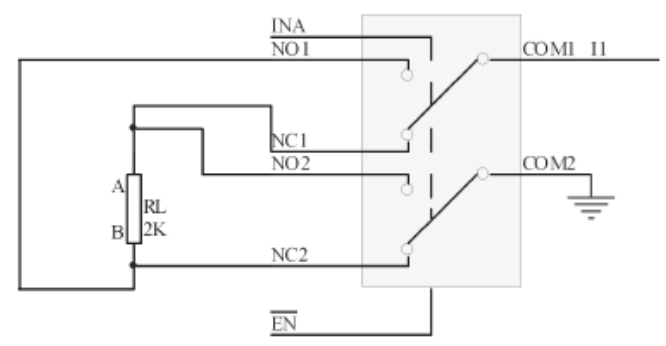

Fig. 3 Connection diagram between analog switch and load resistance

As shown in Fig.3, EN is the key side to activate the chip. INA, connecting with I/O pins of CC2430, has the ability to control the switches. NO1 and $\mathrm{NO} 2$ are normal open pins; $\mathrm{NC} 1$ and $\mathrm{NC} 2$ are normal close pins; COM1 and COM2 are public pins. According to the datasheet of MAX4761A, when voltage of INA is low voltage, NC1 and $\mathrm{NC} 2$ are on, the current whose direction is from $\mathrm{A}$ to $\mathrm{B}$, enters analog switch at COM1 and leaves at COM2; and when voltage of INA is high, NO1 and NO2 are on, the current direction is from $\mathrm{B}$ to $\mathrm{A}$, so both positive and negative pluses to load resistance can be gained. The 
MAX4761A can switch in $\mathrm{nS}$, thus meets the switching speed requirements [8-9].

In the field of animal-robot, power supply is a big problem. Considering the small size of a rat and its limitation of loading, a rechargeable lithium battery weighing $0.8 \mathrm{~g}$ is supplied. The output voltage of lithium battery will drop in the course of working; therefore, in order to ensure that the output voltage is stable at $3.3 \mathrm{~V}$, the regulator is essential. Through a large number of experiments, the conclusion is that positive and negative power supply must be offered to op amp if we want the op amp to work in linear zone. Here MAX660 is used to achieve transformation from $+3.3 \mathrm{~V}$ to $-3.3 \mathrm{~V}$. The output current of MAX660 is about $100 \mathrm{~mA}$, when the output voltage is $-3.3 \mathrm{~V}$, a single MAX660 is able to drive four LM2902s because $0.8 \mathrm{~mA}$ is needed to drive one LM2902. The design of power circuit is shown as Fig.4, MAX1708, having enabling pins, is the regulator, the chip can be switched though the power level added to the 1 and 2 pins. When the chip is functioning, the input voltage is form $0.7 \mathrm{~V}$ to $3.3 \mathrm{~V}$, and the output is stable at $3.3 \mathrm{Vwith}$ maximum current of $2 \mathrm{~A}$.

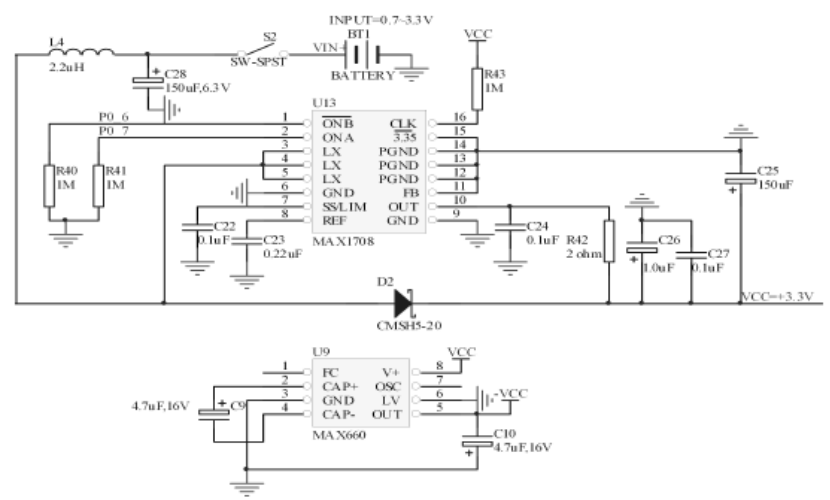

Fig.4 Design of power circuit

\section{Experiments}

The op amp A1 is arranged in saturation zone. PWM frequency is $100 \mathrm{~Hz}$, and the duty cycle is $1 \%$, so the input voltage of $\mathrm{A} 1$ is $+3.3 \mathrm{~V}$, but the output voltage of A1 is $+2.3 \mathrm{~V}$. EDA software Multisim 10 is used as simulation tool, and all resistances and capacitances are with an accuracy of $1 \%$. INA is given a PWM signal with a frequency of $100 \mathrm{~Hz}$, amplitude is $3.3 \mathrm{~V}$. When INA is high, the direction of current is from $\mathrm{B}$ to $\mathrm{A}$, a negative pulse is obtained. When INA is low, NC1 and NC2 are on, the direction of current is from $\mathrm{A}$ to $\mathrm{B}$, a positive pulse is achieved, and the width of pulse is determined by the duty cycle of this PWM. After achieving positive and negative pulse, the enabling pin of MAX4761A is controlled to turn of the analog switches, so that the voltage of load resistance is zero. Simulation result is shown in Fig.5. The cycle of pulse is $10 \mathrm{mS}$ and the time of both positive and negative is $0.2 \mathrm{mS}$. When the input voltage is $+2.3 \mathrm{~V}$ the voltage of load resistance is $460 \mathrm{mV}$, and this result is the same to the theoretical result. In Fig. 5 the burrs with $0.8 \mathrm{nS}$ are generated by analog switches, so the influence can be ignored.

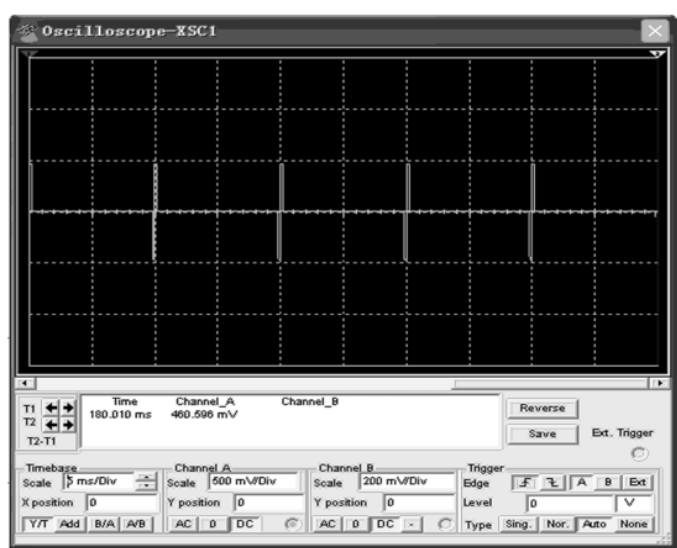

Fig. 5 Result of op amp working in saturation zone

We arrange that the op amp A1 is working in linear zone. PWM frequency is $100 \mathrm{~Hz}$, and the duty cycle is $50 \%$, so the input voltage of $\mathrm{A} 1$ is $+1.65 \mathrm{~V}$, but the output voltage of A1 is $+1.65 \mathrm{~V}$ and other conditions are same as Fig.6. Simulation result is shown in the Fig.6. From the Fig. 6 we can see that if the op amp is working in linear zone when input voltage is $+1.65 \mathrm{~V}$, the voltage of load resistance is $335 \mathrm{mV}$. And the result is same to theoretical result. The influence caused by analog switches can also be ignored.

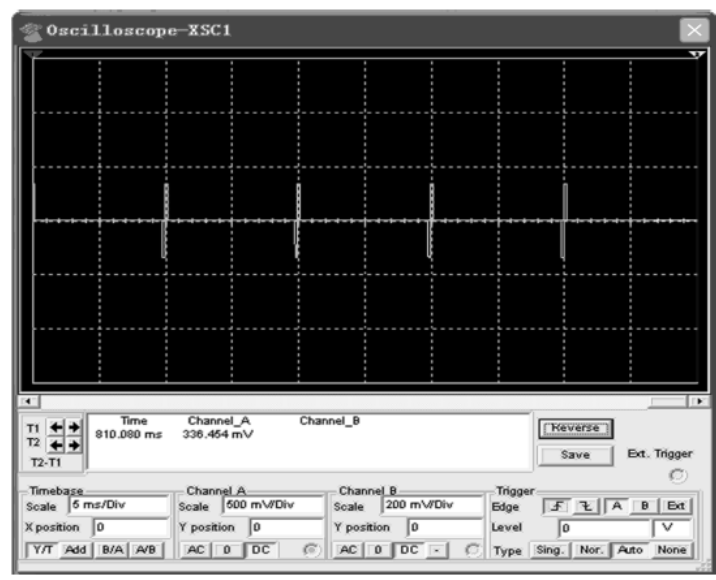

Fig.6 The result of op amp working in linear zone

\section{Summary}

So far we not only have achieved the changes from PWM to DAC but also have successfully solved the problem that the linear zone of op amp is small on the condition it was offered low-voltage power. And the circuit can achieve the output current whose range is form $40 \mathrm{uA}$ to $200 \mathrm{uA}$. We have made some progress about the circuits of animal-robot, but it still cannot achieve requests of fact application, we need further studies. Further study contents include:

(1) How to further reduce weight, and reduce the size?

(2) Because we use CC2430 that supports ZigBee protocol as lower computer, in the next it can be extended that PC is upper computer and CC2430 is the gateway, so 
at the same time we can control sever animal-robots by operating on the upper computer.

(3) At present the way to charge the lithium batteries of animal-robots is still traditional mode that uses charge cable to charge the batteries. If wireless charging the batteries can be achieved it would be greater significance in practice.

\section{Acknowledgments}

The research work was supported by Shandong Province Natural Science Fund No. ZR2014FP004 and Taishan Scholar Establishment Fund.

\section{references}

1. Jr. JB. Ranck. Which elements are excited in electrical stimulation of mammalian central nervous system: a review. Brain Res, 98, 417-420, 1975.

2. E.J. Tehovnik. Electrical stimulation of neural tissue to evoke behavioral responses. Journal of Neuroscience Methods, 65 (1), 1-17, 1996.

3. A.A. Fenton and RU. Muller. Using digital video techniques to identify correlations between behavior and the activity of single neurons. Journal of Neuroscience Methods, 70 (1), 211-215, 1996.

4. I. Obeid, M.A.L. Nicolelis and P.D .Wolf. A multichannel telemetry system for single unit neural recordings. Journal of Neuroscience Methods, 133 (3), 33-37, 2004.

5. J.X. Cai and W.Z. Zhang. Bio-electronics, volume 5 of Progress in Sensor technology and its application, chapter 5, Peking University Press, 22-23, 1997.

6. O.Michio. in: OP amplifier design, edited by L.Wang, volume 3 of Progress in eliminate bias, chapter, 2, Science Press ,2004.

7. M.Lou, C.C. Eschenfelder and T. Herdngen. Therapeutic window for use of hyperbaric oxygenation in focal transient ischemia in rats. Stroke, 35(3), 578-581,2004.

8. Qian Ruzhu. Analysis of voltage controlled constantcurrent sources on operational amplifiers. Electrical Measurement and Instrumentation, 39 (4), 31-33, 2002.

9. Tehovnik E J. Electrical stimulation of neural tissue to evoke behavior responses. Journal of Neuroscience Methods,65(1),1-17,1996. 